\title{
CupAlign: Computer-Assisted Postoperative Radiographic Measurement of Acetabular Components Following Total Hip Arthroplasty
}

\author{
Branislav Jaramaz ${ }^{1,2}$, Constantinos Nikou ${ }^{2}$, Timothy J. Levison ${ }^{1}$, \\ Anthony M. DiGioia III ${ }^{1,2}$, and Richard S. LaBarca ${ }^{2}$ \\ 1 Center for Orthopaedic Research \\ UPMC Shadyside Hospital, Pittsburgh, PA \\ \{branko, levison, digioia\}@cs.cmu.edu \\ http://www. cor.ssh.edu \\ 2 Center for Medical Robotics and Computer Assisted Surgery \\ Carnegie Mellon University, Pittsburgh, PA \\ $\{$ costa,rlabarca\}@ri.cmu.edu \\ http://www.mrcas.ri.cmu.edu
}

\begin{abstract}
Clinical problems following total hip replacement surgery, such as dislocation and implant wear, remain as significant clinical problems with many contributing factors. Although it is intuitive to surgeons that acetabular component alignment is one important factor, large clinical series have produced conflicting results with respect to cup alignment as a risk factor in predisposing to dislocation. One reason is that postoperative measurements of alignment are limited to single or biplanar radiographic measurements. However, the radiographic measurements are limited only to radiographic alignment and may not accurately detail true anatomic orientation of the cup. In this paper, software designed to accurately measure radiographic alignment is described. Postoperative measurements using this system are compared to the actual intraoperative measurements of cup orientation determined by the HipNav ${ }^{\mathrm{TM}}$ imageguided surgical system to assess the accuracy of radiographic evaluation of cup orientation.
\end{abstract}

\section{Introduction}

Orientation of implant system components during total hip replacement (THR) surgery is a key factor in success of the procedure. Implant dislocation following THR ranges between 1 and 5\% [1,2] and represents a significant cause of early failure, incurring additional costs to the total surgery expenses. The causes of dislocation are related to factors such as surgical approach, soft tissue tension, and prosthetic design. One possible dislocation mechanism is the impingement of prosthetic components, in which the implant neck hits the rim of the acetabular liner and levers the head out of the socket[3]. In addition to dislocation, implant impingement causes excessive wear of the cup liner and creation of debris, a 
contributor to implant loosening via bone resorption. Proper alignment of implant components, which is patient and implant specific, reduces the incidence of impingement and reduces the risk of associated complications.

Previous studies have tried to correlate acetabular implant position to dislocation rates. Some researchers have proposed a "safe zone" of cup placement that may lead to minimize the risk of dislocation [4]. Other large clinical studies found no relationship between dislocation and implant placement [5].

Planar radiographs are inexpensive, widely available, and have superior resolution compared with other imaging techniques, and are therefore a preferred tool for post-operative evaluation. This study presents "CupAlign", a new methodology for computer-assisted measurement of cup orientation from radiographs after total hip replacement surgery, and compares its postoperative measurements with intraoperative measurements collected with an image-guided surgical navigation system. The HipNav ${ }^{\mathrm{TM}}$ system [6] provides surgeons with the tools to place the cup precisely within the pelvis and measure its position relative to the pelvic anterior plane (i.e. the plane containing the two anterior iliac spine points and the anterior pubis symphysis points) which is generally oriented vertically when the patient is standing. The HipNav ${ }^{\mathrm{TM}}$ measurement of true anatomic alignment is used in this study to evaluate the results obtained by radiographic techniques for postoperative measurement of cup alignment.

\section{Methods}

During all surgeries in this study, the acetabular cup was placed in the position selected in the preoperative plan using the HipNav ${ }^{\mathrm{TM}}$ image-guided system. Postoperatively, AP radiographs were taken at one and three months. Digitized radiographs are analyzed to reconstruct the position of the cup based on its projected shape using a computer-assisted procedure described below. The measurements are compared to the ones made with the HipNav ${ }^{\mathrm{TM}}$ system, in which the cup orientation is precisely measured intraoperatively, and expressed relative to the pelvic anterior plane.

\subsection{Computer-Assisted Measurement Procedure}

After the radiograph is taken, it is digitized using a flatbed scanner. The digital image is loaded by the cup alignment measurement software (CupAlign). CupAlign allows a surgeon or technician to digitally mark landmarks on the radiographic image using a simple interface (Figure 1).

The first step determines the center of the x-ray beam relative to the radiograph. Metal markers are placed on the patient prior to imaging. The markers denote the central axis of the beam. The distance of the film to the x-ray source is entered to CupAlign as well. This defines the exact position of the x-ray source relative to the radiographic film. With this position, the proper projected images of arbitrary objects in the x-ray beam can be determined. 


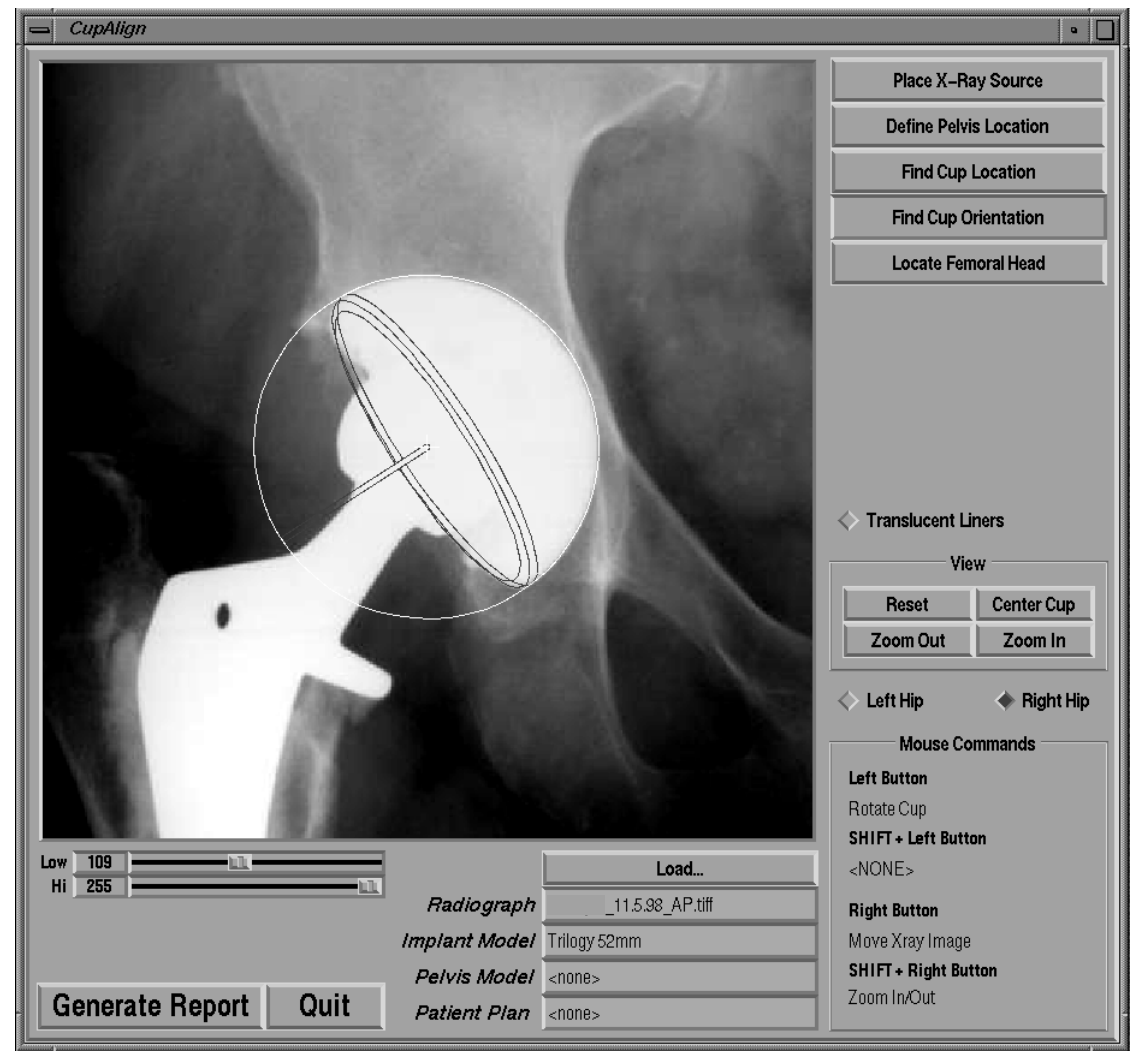

Fig. 1. CupAlign screenshot.

Next, the transverse plane is defined using standard radiographic landmarks of the pelvis, such as the "teardrops". This gives an estimate of rotation of the patient pelvis in the radiographic plane. With that rotation estimate, an object's position relative to the pelvis can be determined, provided the position relative to the radiograph is known.

Since the cup diameter is known, we can then manipulate the position of a virtual sphere with the cup's diameter in radiograph space. CupAlign calculates the projected image of the sphere, and overlays that image on the digitized radiograph (Figure 2). Once the size and shape of the sphere's projection matches the cup on the radiograph, the position of the virtual sphere in radiographic space is the same as the position of the cup during the x-ray imaging.

Once the position of the cup center in space is determined, the user can rotate a model of the cup (from CAD data) about its center until the calculated projection of its characteristic features matches the image in the radiograph (Figure 2). When there is a match, the orientation of the cup relative to the radiograph during the imaging is known. Because we know the position and 
orientation of the pelvis relative to the radiograph as well, we can determine the cup position in the estimated pelvic reference frame.

Once all components are located, CupAlign reports a listing of the relevant data in a text file that can be easily parsed by other software packages. The report includes information describing pelvic abduction relative to the radiograph, $\mathrm{x}-$ ray source location relative to the radiograph, and cup position and orientation relative to the estimated position of the pelvis.

For this study, CupAlign was used to determine the postoperative alignment of the cup in $22 \mathrm{HipNav}^{\mathrm{TM}}$ cases. One technician performed the measurements in all cases to eliminate cross-user measurement error. In order to determine the reliability of orientation information retrieved from standard radiography, the results of the postoperative CupAlign analysis were compared with the actual intraoperative measurements.
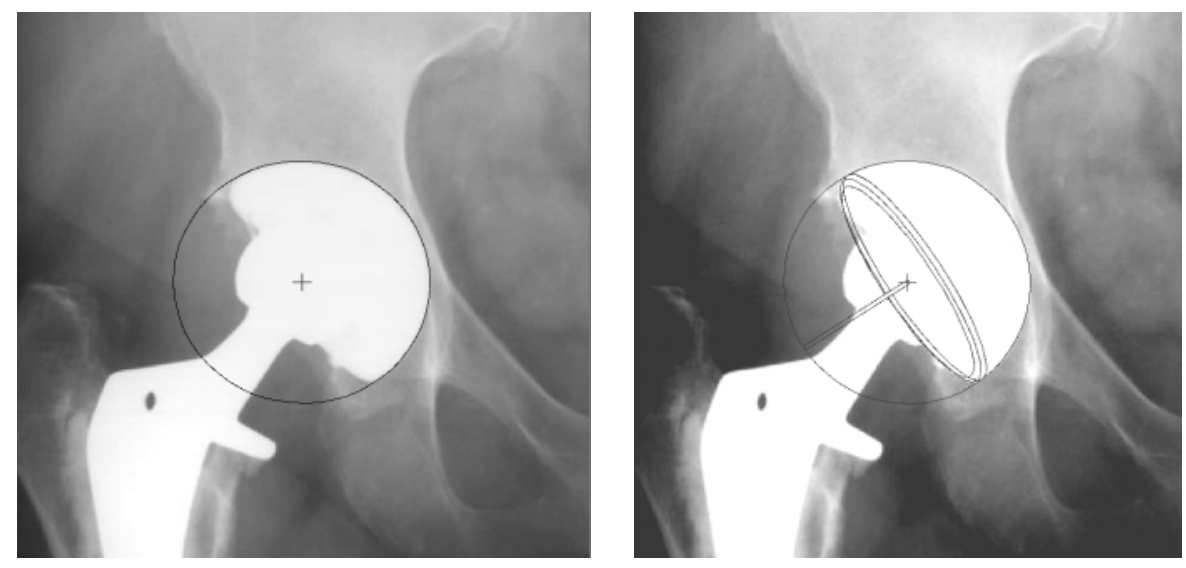

Fig. 2. Placement of the cup in CupAlign. First the position is determined by projecting a sphere with the same size as the cup onto the x-ray film plane and manipulating the location of the sphere (a). The orientation is then determined by matching a projection of a CAD model of the cup's distinguishing features on the image(b).

\section{Results}

Twenty-two of the initial $45 \mathrm{HipNav}^{\mathrm{TM}}$ cases had data including one and three month radiographs available for study. CupAlign was used to determine the postoperative alignment of the cup in all cases. One technician performed the measurements in all cases to eliminate cross-user measurement error.

Figure 3 displays the inter-radiograph position variation. For each case, the $\mathrm{X}$-value of the data point on the graph represents the change in cup flexion, and 


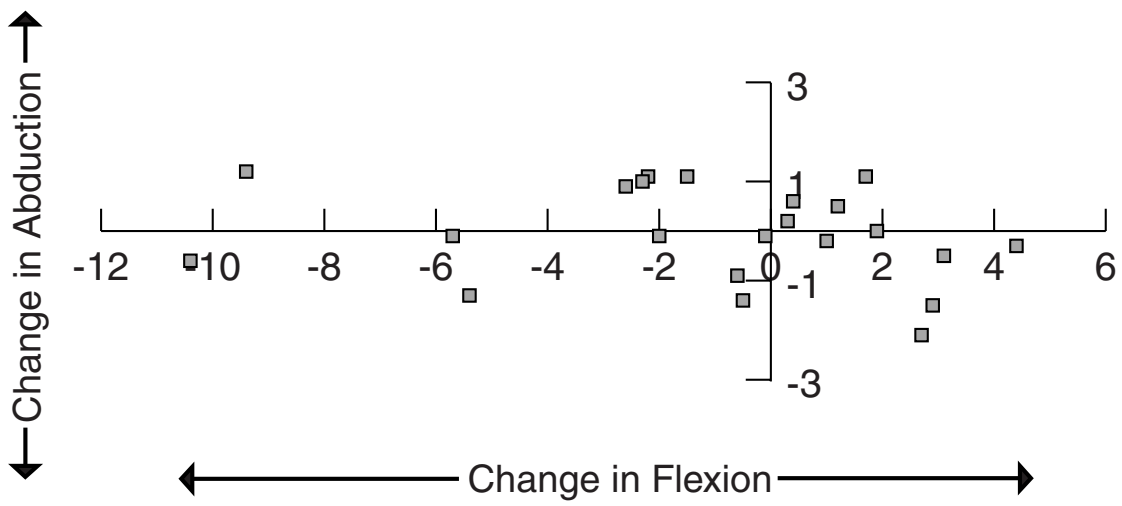

Fig. 3. Change in cup orientation measurement (in degrees) between radiographs taken at one and three months.

the $\mathrm{Y}$-value represents the change in cup abduction. The mean changes in abduction and flexion were $-.1^{\circ}$ and $-1.1^{\circ}$, with standard deviations of $.96^{\circ}$ and $3.85^{\circ}$, respectively.

The variations between intraoperative and radiographic measurements for both the one and three month groups are presented in Figure 4. (The values in the graph are intraoperative values minus the postoperative values). For the onemonth radiographs, the mean change in abduction from the final intraoperative measurement to the CupAlign measurement was $1.2^{\circ}$, with a standard deviation of $3.0^{\circ}$. The flexion change mean was $7.4^{\circ}$ with a standard deviation of $9.4^{\circ}$. Similar results were found when analyzing the three-month radiographs. The abduction changes had a mean of $1.2^{\circ}$ with a standard deviation of $2.8^{\circ}$. The flexion changed an average of $6.5^{\circ}$ with a standard deviation of $8.8^{\circ}$.

\section{Discussion and Future Work}

The minimal change in cup abduction between radiograph measurements is evidence to the repeatability and relative accuracy of abduction measurements with this technique. The variation in cup flexion can be attributed to slight differences in the orientation of the pelvis (flexion and anteversion) relative to the $\mathrm{x}$-ray film from the one month to three month radiographs. Because projection matching is a manual process, human error is also a factor in all measurements.

However, there was a significant variation between intraoperative and all postoperative radiographic measurements for the evaluated cases. Intraoperative measurement of cup orientation with the HipNav ${ }^{\mathrm{TM}}$ system is expressed relative to the pelvic anterior plane, which represents standing neutral pelvic flexion. The variations of cup positions (especially cup flexion) from neutral position are mostly attributed to patient-to-patient differences in pelvic position during imaging. Therefore, without additional controls and measurements of the relative 

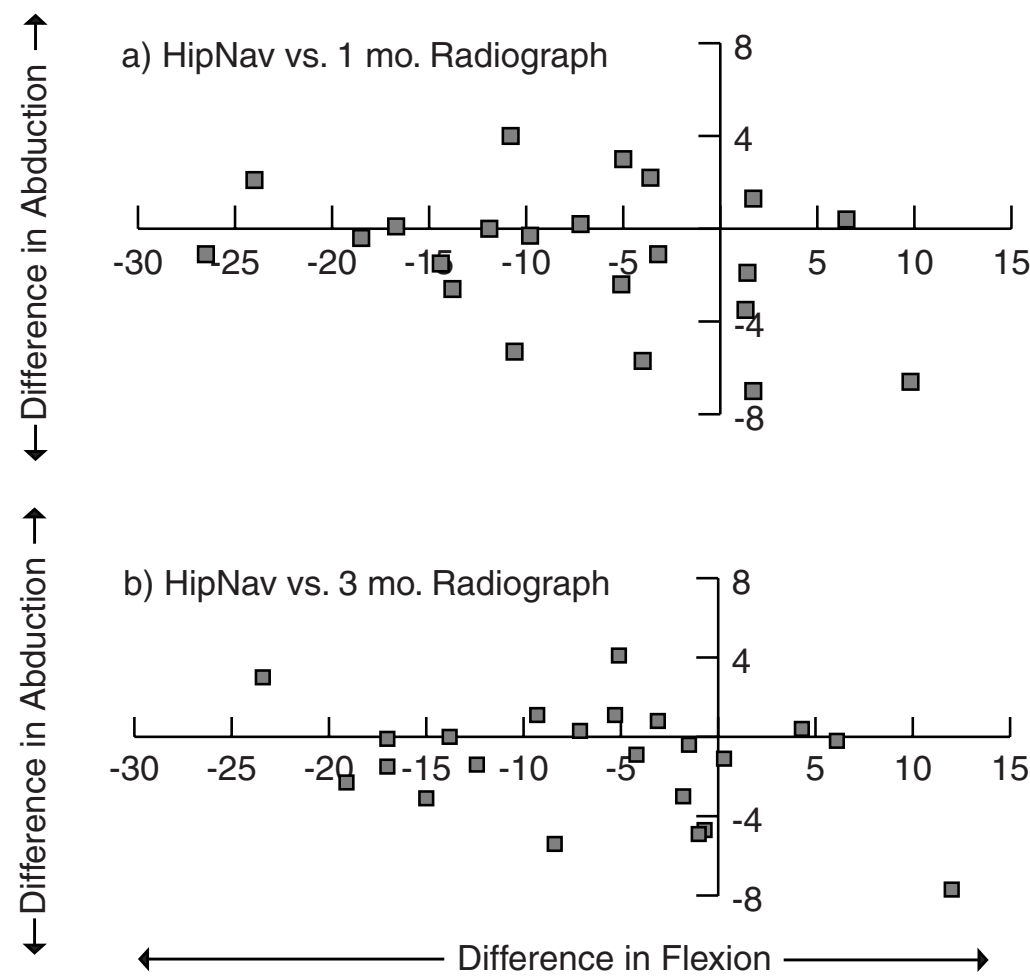

Fig. 4. Difference in cup orientation (in degrees) between intraoperative and postoperative measurements for (a) radiographs one month after surgery and (b) radiographs three months after surgery.

orientation of the pelvis, radiographic measurements are not a reliable method to evaluate cup orientation, especially anteversion alignment. Improvements can be achieved by carefully aligning the neutral plane of the pelvis at the time the radiographs are acquired, or by developing improved techniques for radiographic interpretation of pelvic orientation. Because we measure the cup flexion as well as the more traditional measure of cup anteversion, we can get an estimate of pelvic flexion for each patient, an initial step in understanding functional positions of the pelvis.

In future research, the location of the femoral component head and stem could be calculated in a similar fashion in future versions of CupAlign. Knowledge of postoperative femoral head location relative to the center of the cup liner could be used to estimate wear of the polyethylene liner. Future studies will also address measurement of pelvic position during x-ray acquisition (via automated $2 \mathrm{D}$ to $3 \mathrm{D}$ registration of the pelvis to the radiographic reference frame) and validity of radiographic landmarks. 


\section{References}

1. Cobb, T. K., Morrey B. F., Ilstrup D. M.: The elevated-rim acetabular liner in total hip arthroplasty: Relationship to postoperative dislocation. The Journal of Bone and Joint Surgery, Vol 78-A, No. 1, January 1996, 80-86. 876

2. McCollum, D. E. and Gray, W. J.: Dislocation after total hip arthroplasty (causes and prevention). Clinical Orthopaedics and Related Research 261 (1990): 159-170. 876

3. Amstutz, H. C. and Markolf, K. L.: Design features in total hip replacement. In Harris W.H. (ed.): Proceedings of the Second Open Scientific Meeting of the Hip Society, New York, C.V. Mosby, 1974. 876

4. Lewinnek, G. E., Lewis, J. L., Tarr R., Compere, C. L., Zimmerman, J. R.: Dislocation after total hip-replacement arthroplasties. J. Bone Joint Surg.: 217-220, Vol 60-A, No. 2, March 1978. Clinical Orthopedics and Related Research 354 (1998): 70-81. 877

5. Paterno, S. A., Lachiewicz, M. D., and Kelley, S. S: The Influence of Patient-Related Factors and the Position of the Acetabular Component on the Rate of Dislocation After Total Hip Replacement. The Journal of Bone and Joint Surgery, Vol 78-A, No. 8, August 1997, 1202-1210. 877

6. DiGioia et al.: Image Guided Navigation System to Measure Intraoperatively Acetabular Implant Alignment. Clinical Orthopaedics and Related Research 355 (1998): 8-23. 877 